\title{
Venous thromboembolism in malignancy
}

\author{
Authors: Anna Mullard ${ }^{A}$ and Helen Innes ${ }^{B}$
}

\section{Introduction}

Venous thromboembolism (VTE) is an important cause of morbidity and mortality in patients with cancer. It occurs in up to $20 \%$ of patients with an overt malignancy and is the second leading cause of death in these patients. ${ }^{1}$ Complex cancer- and treatment-related symptoms can mask VTE symptoms, leading to atypical presentations, such as progressive dyspnoea. Therefore, clinicians should have a low threshold of suspicion for both deep venous thrombosis (DVT) and pulmonary embolism (PE) in any patient with cancer. VTE can also precede the diagnosis of cancer by many months; therefore, any unprovoked VTE in someone over 40 years of age should prompt investigations into the possibility of an occult malignancy. ${ }^{2}$

\section{Hypercoagulable state}

VTE is the most common thrombotic condition associated with malignancy, but others can occur: marantic endocarditis, disseminated intravascular coagulation, arterial thrombosis, thrombotic microangiopathy and migratory superficial thrombophlebitis are all described. There is general agreement that patients with cancer are in a hypercoagulable state. Virchow's triad suggests that VTE occurs because of three factors: alteration in blood flow, vascular endothelial injury and alteration in the constituents of the blood. Turbulent blood flow can occur because of extrinsic compression from malignant tumours or intrinsic devices, such as central venous catheters. Vascular endothelial injury can be induced by systemic anticancer therapies. The prothrombotic effect of antiangiogenic agents, such as bevacizumab and thalidomide, are particularly high. Procoagulants released from cancer and normal cells along with a decrease in natural coagulation inhibitors (reduced levels of proteins C and S) and increased platelet activation can also contribute. ${ }^{3}$

Certain types of cancer confer a particularly high risk. Cancers of the pancreas, brain and liver, in addition to multiple myeloma, are cited as having the highest incidence. ${ }^{4}$ Patients with cancer might also share many of the factors that predispose noncancer patients to VTE (Box 1).

Authors: AST6 medical oncology, Betsi Cadwaladr University Health

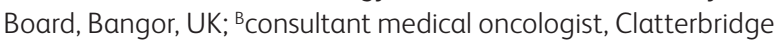
Cancer Centre NHS Foundation Trust, Wirral, UK
Box 1. Risk factors for venous thromboembolism

in patients with malignant disease.

\section{Cancer-related risk factors}

$>$ Primary site of cancer

Extensive disease

Systemic anticancer therapy (chemotherapy, novel agents or hormonal manipulation)

> Presence of central venous catheter

$>$ Steroid treatment

$>$ Recent surgery

Haematological abnormalities (high platelet count, low haemoglobin or high white cell count)

> Time after initial diagnosis (first 6 months are highest risk)

\section{Patient-related risk factors}

Raised body mass index

Reduced mobility

$>$ Comorbidities (respiratory disease and/or renal disease)

Older age

> Previous history of venous thromboembolism

Sepsis

Ethnicity (increased in those of black African descent)

\section{Management}

In general, the initial management of confirmed VTE comprises supportive care and immediate anticoagulation, regardless of cancer diagnosis. However, the increased likelihood of contraindications to immediate anticoagulation should be considered at an early stage (Box 2).

Whereas noncancer patients are usually switched to an oral anticoagulant (most commonly warfarin) as soon as possible, this is not best practice for patients with a known malignancy. Unstable international normalised ratio (INR) levels as a result of changeable nutrition, liver function and drug interactions often makes monitoring troublesome and risks both sub- and supertherapeutic levels. Low-molecular-weight heparin (LMWH) reduces the recurrence rate of VTE without increasing the risk of bleeding and is recommended for at least 6 months in patients with cancer in the absence of any known contraindication (Box 2)..$^{2,5,6}$ 
Box 2. Contraindications to therapeutic low molecular weight heparin (LWMH).

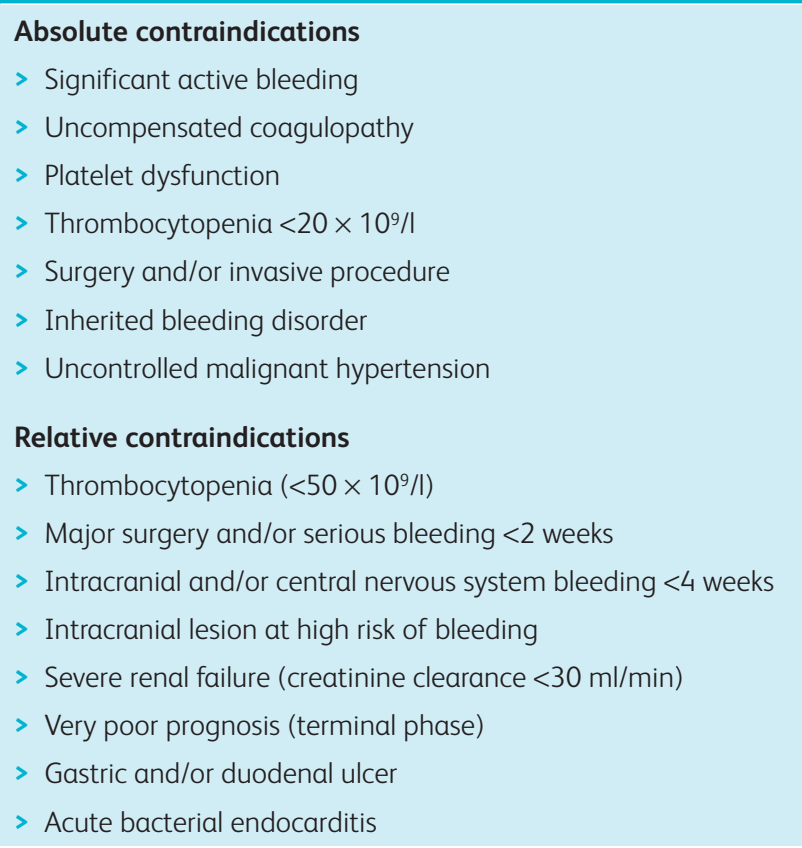

Patients with severe renal impairment (estimated glomerular filtration rate $<30 \mathrm{ml} / \mathrm{min}$ ) should be treated with unfractionated heparin. ${ }^{2}$ However, continued treatment with reduced dose LMWH and anti-factor Xa monitoring might be more convenient if renal function is unlikely to improve.

There are no robust data to support the use of LMWH beyond 6 months in patients with cancer and a proven VTE; however, patients with ongoing malignancy remain at risk; therefore, careful consideration of indefinite treatment is advisable. ${ }^{3}$

\section{Key points}

Patients with cancer are at high risk of venous thromboembolism (VTE)

The potential for atypical VTE presentations in patients with cancer should prompt a low threshold for investigation

Prophylactic anticoagulation is recommended for surgical and medical inpatients with a malignancy

Low molecular-weight heparin (LMWH) is the optimal treatment for patients with cancer with a proven VTE

Incidental VTE in a patient with cancer requires immediate assessment and management regardless of whether it is symptomatic or not

KEYWORDS: Venous thromboembolism, malignancy, lowmolecular-weight heparin, primary prophylaxis, incidental a
Patients with underlying malignancy and VTE are underrepresented in studies of novel oral anticoagulants (NOAC). This, combined with concerns around the lack of antidote for these agents, means their use cannot be recommended and should be reserved for use within a clinical trial.

Life- or limb-threatening VTE remain rare. PE associated with right ventricular dysfunction or an extensive DVT that threatens limb perfusion are examples of cases requiring assessment for suitability of thrombolytic therapy. Prompt thrombolysis with agents such as streptokinase can achieve rapid clot breakdown and quickly restore perfusion.

Most patients with cancer and VTE remain well and common sense dictates that these patients can be rapidly discharged back to community services. Informing the local acute oncology team of the admission will help to ensure that the relevant specialist teams are kept up to date and that continuity of care is maintained.

\section{Recurrent VTE}

Recurrent VTE despite anticoagulation occurs in approximately $9 \%$ of patients taking $\mathrm{LMWH}$ and $17 \%$ of patients taking oral anticoagulation, and should prompt investigation into compliance and/or adequacy of initial treatment. ${ }^{7}$ Any patient taking an oral anticoagulant should be switched to LMWH or unfractionated heparin where possible. By contrast, those patients already taking LMWH should have their dose:weight ratio optimised. Cases of VTE recurrence despite therapeutic doses of LMWH can be considered for vena cava filter or dose-escalated LMWH where appropriate. However, vena cava filters remain controversial. They have shown a reduction in PE in patients with VTE (both with or without cancer), but with an increase in DVT and no difference in patient survival. ${ }^{8}$ These filters are often used in cases where anticoagulation is not possible; however, they should ideally be used alongside therapeutic anticoagulation. Although dose-escalated LMWH (20-25\% increase) is cited within international guidelines as an option for recurrent VTE, this is based on retrospective data. ${ }^{9}$

\section{Incidental VTE}

Fears that therapeutic anticoagulation for incidental PE might constitute overtreatment appear unfounded. Reports suggest no difference in distribution, recurrence or mortality of PEs between symptomatic and incidental cases. ${ }^{10}$ As a result, the current consensus is that incidental PEs should be treated with the same urgency as their symptomatic counterparts. With increasing numbers of patients with cancer receiving outpatient care, this can present a logistical problem to clinicians in locating, assessing, informing and treating these patients in a timely fashion. Ideally, a clear pathway for such patients should be negotiated between radiology, acute oncology, medical and primary care teams, enabling appropriate patients to remain at home. ${ }^{11}$

\section{Prevention}

Despite clearly defined risk factors for VTE in malignancy, there are insufficient data to support pharmacological VTE prophylaxis in ambulant patients with cancer. This is reflected in both national and international guidelines, where 
primary prophylaxis is not recommended because of the lack of significant impact on patient survival., ${ }^{3,12,13}$ Patients with multiple myeloma who are receiving thalidomide and/or lenalidomide-based chemotherapy and/or dexamethasone regimens are the exception to this rule. Their risk of serious thromboembolic events (acute cardiovascular event or sudden death) is $5-8.2 \%$ during the first 6 months of treatment; therefore, primary prophylaxis with aspirin, warfarin or LMWH is recommended. ${ }^{14}$

Once hospitalised, primary prophylaxis is advisable because of the high risk of VTE in immobile patients. As a result, pharmacological prophylaxis with LMWH, UFH or fondaparinux is indicated. ${ }^{3,5}$ Patients with cancer undergoing major surgery should also ideally receive primary prophylaxis starting preoperatively and continuing for up to 7-10 days postoperatively., ${ }^{3,13}$ Mechanical thromboprophylaxis alone is felt to be insufficient for patients with cancer and is used in cases where pharmacological measures are not possible. For those patients undergoing major abdominal or pelvic surgery, extended pharmacological prophylaxis is indicated for up to 4 weeks postoperatively because of the prolonged high incidence of VTE..$^{15}$

\section{References}

1 Lyman GH, Khorana AA, Falanga A et al. American Society of Clinical Oncology Guideline: recommendations for venous thromboembolism prophylaxis and treatment in patients with cancer. J Clin Oncol 2007;25:5490-505.

2 National Institute for Health and Care Excellence. Venous thromboembolic disease: the management of venous thromboembolism diseases and the role of thrombophilia testing. London: NICE, 2010. www.nice.org.uk/guidance/cg144 [Accessed 5 August 2014].

3 Mandala M, Falanga A, Roila F. Management of venous thromboembolism (VTE) in cancer patients: ESMO Clinical Practice Guidelines. Ann Oncol 2011;22(Suppl 6):vi85-vi92.

4 Cronin-Fenton DP, Sondergaard F, Pederson LA et al. Hospitalisation for venous thromboembolism in cancer patients and the general population: a population-based cohort study in Denmark, 1997-2006. Br J Cancer 2010;103:947.

5 Scottish Intercollegiate Guidelines Network. Prevention and management of venous thromboembolism. Quick Reference Guide 122. Edinburgh: Scottish Intercollegiate Guidelines Network, 2010. www.sign.ac.uk/pdf/qrg122.pdf [Accessed 5 August 2014].
6 Akl EA, Labedi N, Barba $\mathrm{M}$ et al. Anticoagulation for the long-term treatment of venous thromboembolism in patients with cancer. Cochrane Database Syst Rev 2011;6:CD006650.

7 Lee Ay, Levine MN, Baker RI et al. Randomised comparison of lowmolecular-weight heparin versus oral anticoagulant therapy for the prevention of recurrent venous thromboembolism in patients with cancer (CLOT) investigators. Low-molecular-weight heparin versus a coumarin for the prevention of recurrent venous thromboembolism in patients with cancer. N Engl J Med 2003;349:146-53.

8 PREPIC Study Group. Eight year follow-up of patients with permanent vena cava filters in the prevention of pulmonary embolism: the PREPIC (Prevention du Risque d'Embolie Pulmonaire par Interruption Cave) randomised study. Circulation 2005;112:416-22.

9 Carrier M, Le Gal G, Cho R et al. Dose escalation of low molecular weight heparin to manage recurrent venous thromboembolic events despite systemic anticoagulation in cancer patients. J Thromb Haemost 2009;7:760-5.

10 Palla A, Rossi G, Falaschi F et al. Is incidentally detected pulmonary embolism in cancer patients less severe? A case-control study. Cancer Invest 2012;30:131-4.

11 Royal College of Physicians. Acute Care Toolkit 7: Acute oncology on the acute medical unit. London: RCP, 2013. www.rcplondon.ac.uk/ sites/default/files/acute_care_toolkit_7.pdf [Accessed 5 August 2014].

12 National Institute for Health and Care Excellence. Reducing the risk of venous thromboembolism (deep vein thrombosis and pulmonary embolus) in patients admitted to hospital. London: NICE, 2010. www.nice.org.uk/guidance/cg92 [Accessed 5 August 2014].

13 Lyman GH, Khorana AA, Kuderer NM et al. Venous thromboembolism prophylaxis and treatment in patients with cancer: American Society of Clinical Oncology Clinical Practice Guideline Update. J Clin Oncol 2013;31:2189-204.

14 Palumbo A, Cavo M, Bringhen S et al. Aspirin, warfarin or enoxaparin thromboprophylaxis in patients with multiple myeloma treated with thalidomide: a phase III, open-label, randomised trial. J Clin Oncol 2011;29:986-93.

15 Akl EA, Terrenato I, Barba $\mathrm{M}$ et al. Extended perioperative thromboprophylaxis in patients with cancer: a systematic review. Thromb Haemost 2008;100:1176-80.

Address for correspondence: Dr H Innes, Clatterbridge Cancer Centre NHS Foundation Trust, Clatterbridge Road, Bebington, Wirral CH63 4JY.

Email: helen.innes@clatterbridgecc.nhs.uk 